ARTICLE

\title{
Designer spin order in diradical nanographenes
}

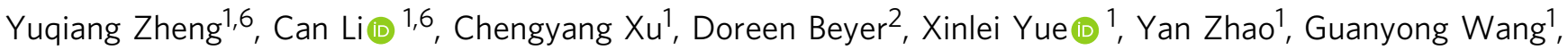

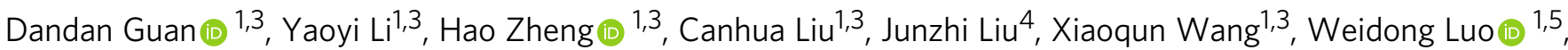 \\ Xinliang Feng (i) ${ }^{2 凶}$, Shiyong Wang (1) ${ }^{1,3 凶} \&$ Jinfeng Jia (i) ${ }^{1,3 凶}$
}

The magnetic properties of carbon materials are at present the focus of intense research effort in physics, chemistry and materials science due to their potential applications in spintronics and quantum computing. Although the presence of spins in open-shell nanographenes has recently been confirmed, the ability to control magnetic coupling sign has remained elusive but highly desirable. Here, we demonstrate an effective approach of engineering magnetic ground states in atomically precise open-shell bipartite/nonbipartite nanographenes using combined scanning probe techniques and mean-field Hubbard model calculations. The magnetic coupling sign between two spins was controlled via breaking bipartite lattice symmetry of nanographenes. In addition, the exchange-interaction strength between two spins has been widely tuned by finely tailoring their spin density overlap, realizing a large exchange-interaction strength of $42 \mathrm{meV}$. Our demonstrated method provides ample opportunities for designer above-room-temperature magnetic phases and functionalities in graphene nanomaterials.

\footnotetext{
${ }^{1}$ Key Laboratory of Artificial Structures and Quantum Control (Ministry of Education), Shenyang National Laboratory for Materials Science, School of Physics and Astronomy, Shanghai Jiao Tong University, 200240 Shanghai, China. ${ }^{2}$ Center for Advancing Electronics Dresden and Department of Chemistry and Food Chemistry, Technische Universität Dresden, 01062 Dresden, Germany. ${ }^{3}$ Tsung-Dao Lee Institute, Shanghai Jiao Tong University, 200240 Shanghai, China. ${ }^{4}$ Department of Chemistry and State Key Laboratory of Synthetic Chemistry, The University of Hong Kong, Pokfulam Road, Hong Kong, China. ${ }^{5}$ Institute of Natural Sciences, Shanghai Jiao Tong University, 200240 Shanghai, China. ${ }^{6}$ These authors contributed equally: Yuqiang Zheng, Can Li. 巴email: xinliang.feng@tu-dresden.de; shiyong.wang@sjtu.edu.cn; jfjia@sjtu.edu.cn
} 
$\mathrm{N}$ anographenes (NGs) with certain $\pi$-electron topologies are predicated to host manifold magnetic properties, promising for designer miniaturized spin-based devices and for quantum computations ${ }^{1-15}$. The study of magnetic nature of NGs traces back to 1950s, where Clar established an empirical method to determine the number of unpaired electrons in NGs by drawing non-kekule structure ${ }^{16}$. A typical example is the bowtieshaped NG, also called Clar goblet, which holds two unpaired electrons with an antiferromagnetic coupled ground state ${ }^{8,17}$. In 1989, Lieb came up with a theorem for bipartite lattice stating that NGs with sublattice imbalance host a net spin of $S=$ $\left(N_{A}-N_{B}\right) / 2$ with $N_{A} / N_{B}$ denoting the number of carbon atoms in each triangular sublattice $A / B$ of graphene honeycomb lattice ${ }^{3}$. Very recently, Ortiz et al studied the exchange interactions of bipartite NGs by using a set of combined theoretical methods at different levels to determine both the ground state and the first few excited states ${ }^{18}$. They found that magnetic exchange of diradical bipartite NGs depends crucially on sublattice imbalance, point symmetry group, and wavefunction overlap of zero modes.

Departing from the above bipartite NGs, certain NGs with non-hexagonal rings, also called nonbipartite NGs, host nontrivial magnetic features and have seldomly been addressed. Previous theory calculations demonstrated that incorporation of carbon tetragons/pentagons in zigzag graphene nanoribbons switches the spin orientation at each edge, and such spin switches can lift the degeneracy between the two spin propagation channels ${ }^{13,14}$. Since magnetic NGs are usually unstable and reactive due to the presence of radicals, the fabrication of unsubstituted NGs has remained elusive for a long time, although the addition of substituents has allowed the solution synthesis of few openshell NG cores and verification of their magnetic ground state via electron paramagnetic resonance measurements ${ }^{19}$. Recent advances in on-surface chemistry have made it possible to fabricate reactive graphene nanostructures with atomic precision and unprecedented tunability by using small molecules as building blocks, with examples of Clar goblet ${ }^{17}$, zigzag graphene nanoribbons ${ }^{20,21}$, Chiral graphene nanoribbons ${ }^{22,23}$ and NGs with sublattice imbalance ${ }^{24-29}$. As demonsrated by $\mathrm{Li}$ et al., the incorporation of pentagon rings in chiral graphene nanoribbons introduces one unpaired electron and two nearby spins can antiferromagnetic coupled together ${ }^{23}$. We recently demonstrated that nanographenes with such pentagon rings also host net spins and the coupling strength can be engineered by tailoring spin density overlap at the connecting region ${ }^{30}$.

Here, we further demonstrate a generic approach to control spin orders inside diradical NGs. Incorporation of a pentagon into a magnetic diradical NG drives the ferromagnetic coupled ground state into antiferromagnetic coupled ground state. The nature behind this exchange interaction, as we have revealed, is a local reversal of spin density sign at the connecting region of the dimer via breaking the bipartite lattice symmetry of NGs. This exchange mechanism is expected to be generic and widespread, because it relies solely on very general features of the nonbipartite character of NGs. Moreover, since it is feasible to precisely tailor the chemical structure of NGs by using on-surface synthesis, the established approach can be extended to design any artificial quantum spin systems, opening wide possibility for fundamental research and applications in spintronics and quantum information technologies.

\section{Results}

Mean-field Hubbard model calculations on diradical nanographenes. Figure 1 provides a paradigm of such exchange mechanism. The bipartite single radical NG considered (cf. Fig. 1a) hosts 14 carbon atoms in $A$ sublattice and 15 carbon atoms in $B$ sublattice, as marked by hollowed and solid spheres, respectively. As per Lieb's theorem, this NG should host a single spin of $S=1 / 2$ due to sublattice imbalance. Mean-field Hubbard model and spin-polarized density functional theory (SP-DFT) calculations also confirm the presence of one unpaired electron with enhanced spin density distribution at the right side (cf. Fig. $1 \mathrm{~b}$ and Supplementary Figs. 3, 4). Figure 1d depicts a nonbipartite NG, which is similar as the above bipartite NG but with a single $\mathrm{C}-\mathrm{C}$ bond, as marked by the red line, connecting two carbon atoms belonging to the same sublattice. The addition of this bond introduces a pentagon ring into the NG, and breaks the bipartite lattice symmetry. We refer to the bipartite NG and the nonbipartite NG as BNG and nBNG hereinafter, respectively. The nBNG also hosts a single spin of $S=1 / 2$ as confirmed by SP-DFT and Hubbard model calculations (cf. Fig. 1g). The overall spin density distribution of $\mathrm{nBNG}$ is similar as that of BNG with enhanced spin density at the right side. However, the spin density distribution at the left terminal phenyl ring of $\mathrm{nBNG}$, as marked by dashed squares, is drastically different from that of BNG, where the sign of spin density gets reversed at this phenyl group as a result of the presence of the red $\mathrm{C}-\mathrm{C}$ bond.

To reveal the effects of spin density sign reversal in the nBNG on the spin exchange interaction, the ground states of different NG dimer configurations have been determined. Figure 1c depicts a BNG-BNG dimer with 28 carbon atoms in $A$ sublattice and 30 carbon atoms in $B$ sublattice, hosting a ferromagnetic coupled ground state of $S=1$ as per Lieb's theorem. The two coupled spins have a magnetic exchange-interaction strength of $7 \mathrm{meV}$ as obtained by Hubbard model calculations. In sharp contrast to the diradical BNG-BNG dimer, the BNG-nBNG dimer in Fig. If hosts an antiferromagnetic coupled ground state with $S=0$. We attribute the transition from ferromagnetic coupled ground state in the BNG-BNG dimer to antiferromagnetic coupled ground state in the BNG-nBNG dimer to the presence of on-site Coulomb repulsion. Since each carbon atom provides a $P_{z}$ orbital and one $\pi$ electron, NGs are ideal two-dimensional half-filling systems. At half filling, spin up density prefers to populate at one sublattice while spin down density at another in order to minimize the on-site Coulomb repulsion (to prevent a double occupation of one $P_{z}$ orbital at the same carbon site) ${ }^{3}$. Since the spin density sign at the connecting region is reversed at the $\mathrm{nBNG}$ side in the dimer, the spin exchange sign will also get reversed to minimize the on-site Coulomb repulsion (Otherwise, the two adjacent carbon sites at the connecting region will host the same spin density sign, driving the system to a higher energy due to onsite Coulomb repulsion). Additional calculations have been made on other configurations of BNG-BNG dimers, $\mathrm{nBNG}-\mathrm{nBNG}$ dimers, nBNG-BNG dimers, and BNG-nBNG dimers (cf. Supplementary Fig. 5). All the configurations agree with the previous physics picture: once the spin density sign gets reversed at the connecting region, the sign of magnetic exchange between the two spins will also get reversed. This unique property of nBNG provides a generic approach to control spin order in $\mathrm{nBNG}$.

Kondo resonances of nanographenes with $S=\mathbf{1 / 2}$. We demonstrate the proposed magnetic exchange mechanism for diradical NGs experimentally. Figure 2a, b depict the chemical structure and nc-AFM image of on-surface synthesized BNG monomers. The BNG hosts a methyl group which exhibits as a bright protrusion in nc-AFM imaging. As confirmed by our DFT calculations, the presence of methyl group does not affect the magnetic properties compared to the counterpart without a methyl group (cf. Supplementary Fig. 4). In addition, the magnetic nanographenes are embedded in a non-magnetic polymer 


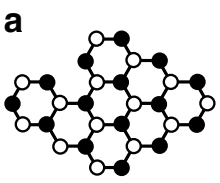

BNG

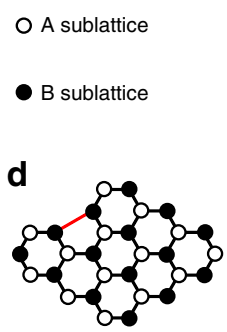

nBNG b

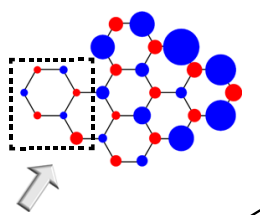

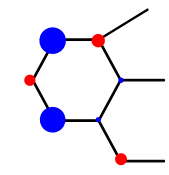

e

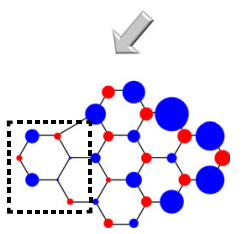

C

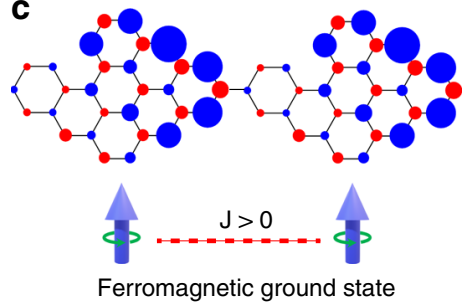

Ferromagnetic ground state

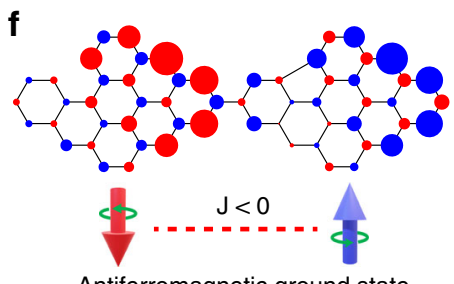

Antiferromagnetic ground state

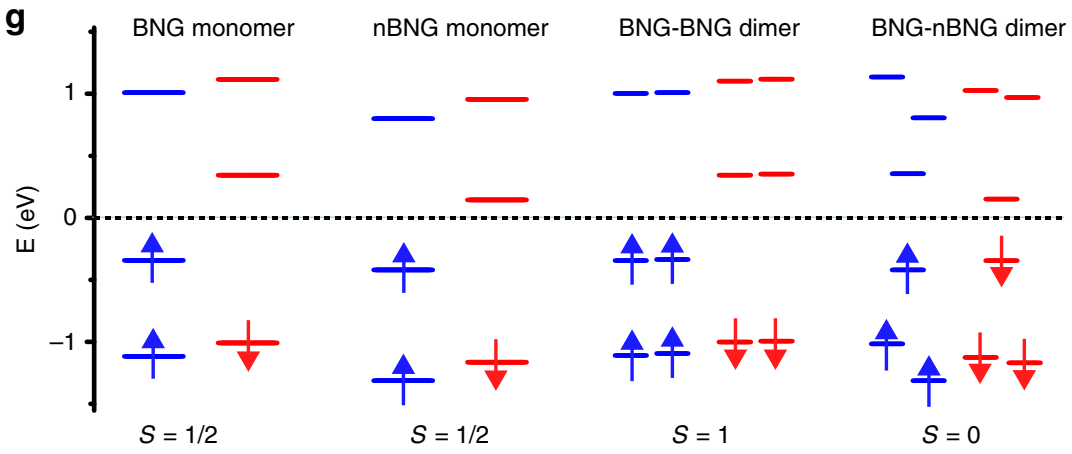

Fig. 1 Controlling spin order in diradical nanographenes via breaking sublattice symmetry. a, Atomic structure of a rhombic bipartite nanographene with a net spin of $S=1 / 2$ due to sublattice imbalance. $\mathbf{b}$ Spin density distribution of the nanographene in $\mathbf{a}$. c Spin density distribution of a diradical BNG-BNG dimer, exhibiting a ferromagnetic coupled ground state. $\mathbf{d}$ Atomic structure of a nonbipartite nanographene with the red $\mathrm{C}-\mathrm{C}$ bond breaking the bipartite sublattice symmetry of nanographenes. The nonbipartite nanographene also hosts a net spin of $S=1 / 2$. e Spin density distribution of the nanographene in d. The presence of five-member ring causes a spin density sign reversal near the red bond, as highlighted by a dashed box. $\mathbf{f}$ Spin density distribution of a diradical BNG-nBNG dimer, showing an antiferromagnetic coupled ground state. $\mathbf{g}$ Energy level filling of the above four configurations. Blue/red isosurfaces denote spin up/spin down density, respectively.

(cf. Supplementary Fig. 1). As confirmed theoretically and experimentally, the presence of neighboring non-magnetic units does not affect the magnetic properties of embedded magnetic nanographenes (cf. Supplementary Figs. 6 and 8). The presence of a net spin of $S=1 / 2$ in the bipartite NG is confirmed by Kondo resonance, which gives a sharp peak at Fermi level in differential conductance $(\mathrm{d} I / \mathrm{d} V)$ spectra. The Kondo resonance originates from the coupling of the magnetic impurity by $\mathrm{Au}(111)$ electron reservoir, and can be understood by single impurity Anderson Model $^{31,32}$. This model gives a temperature dependent Kondo peak width with full width at half maximum (FWHM) of $\Gamma=\sqrt{\left(\alpha k_{B} T\right)^{2}+\left(2 k_{B} T_{K}\right)^{2}}$, where $T$ is the temperature, $T_{K}$ is the Kondo temperature, and $\alpha$ is the slope of linear growth of the width at $T \gg T_{K}$. The experimentally obtained temperature dependent FWHM in Fig. $2 \mathrm{~h}$ can be well fitted by the previous equation, giving a Kondo temperature of $39 \mathrm{~K}$. The Kondo peak is expected to response to an external magnetic field, and start to split for magnetic field up to $B_{C} \approx 0.5 K_{B} T_{K} /\left(g \mu_{B}\right)^{32}$. The threshold magnetic field for Kondo peak splitting in our case is estimated to be $15 \mathrm{~T}$, which is beyond the capability $(10 \mathrm{~T})$ of our system (We notice the Kondo peak becoming broadened as increasing magnetic field, but we did not observe Kondo peak splitting for magnetic field up to $9.5 \mathrm{~T}$ (cf. Fig. 2f)). The net single spin in $\mathrm{nBNG}$ monomers has also been carefully studied ${ }^{30}$, and similar observations have been obtained (cf. Supplementary Fig. 7).

Spin flip spectroscopy of diradical nanographenes. The magnetic exchange for diradical NG dimers has been detected by spin flip spectroscopy, where inelastic tunneling electrons can excite the ground state into an excited state when the energy of tunneling electrons exceeds the energy difference between the two states $^{17,23,33-35}$. The chemical structure and nc-AFM image of an achieved BNG-BNG dimer is depicted in Fig. 3a. In contrast to a sharp resonance obtained on the BNG monomer, $\mathrm{d} I / \mathrm{d} V$ spectra made on a BNG-BNG dimer exhibits three resonances, with a sharp peak at fermi level and two side shallow shoulders at $-7 \mathrm{meV}$ and $7 \mathrm{meV}$, respectively. These features originate from the screening of a net spin of $S=1$ by $\mathrm{Au}(111)$ surface electrons (zero energy Kondo peak) and spin-flip excitations (two side peaks) from ground triplet state to excited singlet state. From the spin-flip feature, we can directly obtain the magnetic exchangecoupling strength of $7 \mathrm{meV}$, which agrees with Hubbard model calculations $(7.7 \mathrm{meV})$. To qualitatively capture the scattering process inside an STM junction, we simulate our $\mathrm{d} I / \mathrm{d} V$ spectra using a perturbative approach up to third order using codes provided by Ternes ${ }^{36}$, where the simulated spectra nicely reproduce the experimental features (cf. Supplementary Fig. 9). 
a

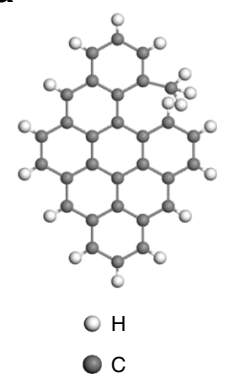

e

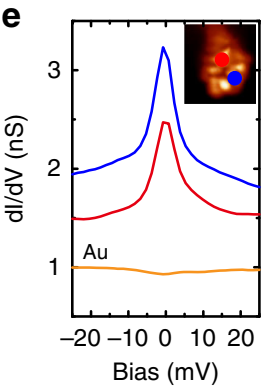

b

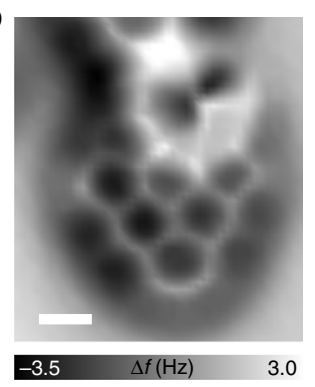

$\mathbf{f}$

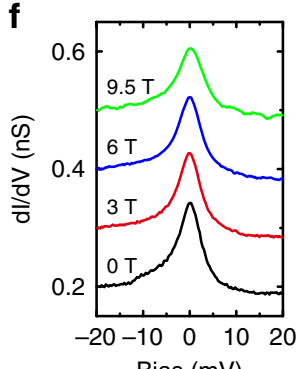

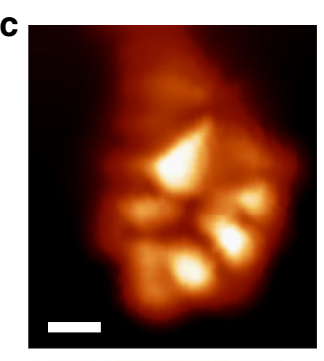
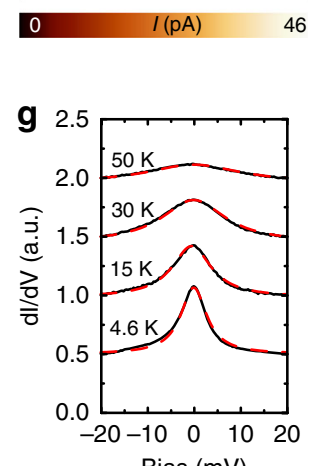
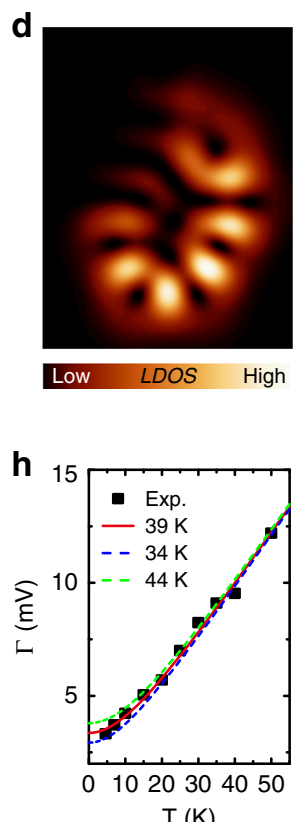

Fig. 2 Detecting the net spin in a bipartite nanographene monomer via Kondo resonance. a Chemical structure of a rhombic bipartite nanographene. b Nc-AFM frequency shift image (Resonant frequency: $29 \mathrm{KHz}$, Oscillation amplitude: 160 pm) of the nanographene in a. The methyl group is imaged

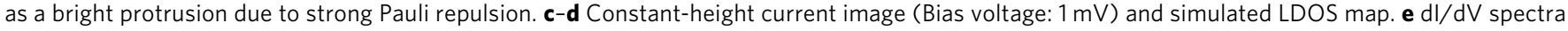
taken on the two locations marked on the inset current image. A sharp zero-energy peak is resolved due to the screening of the net spin of $S=1 / 2$ in nanographene by $\mathrm{Au}(111)$ surface electrons. f Out-of-plane magnetic field dependence of the Kondo resonance. All $\mathrm{d} l / \mathrm{d} V$ spectra were taken at the same position. $\mathbf{g}$ Temperature dependence of the Kondo resonance. All $\mathrm{d} l / \mathrm{d} V$ spectra were taken at the same position. The dotted lines are simulated curves using a Frota function. $\mathbf{h}$ The full peak width at half maximum as a function of temperature. A Kondo temperature of $39 \mathrm{~K}$ is obtained. Scale bars: $0.3 \mathrm{~nm}$.

Figure $3 \mathrm{~d}$ depicts the chemical structure and nc-AFM image of a BNG-nBNG (from up to down) hetero-dimer. In sharp contrast to BNG-BNG dimer, $\mathrm{d} I / \mathrm{d} V$ spectra exhibit two symmetric steps at both positive and negative bias, indicating an antiferromagnetic ground state with $S=0$. The two steps at the same bias amplitude are due to spin excitation from the ground singlet state to excited triplet state, giving an exchange-coupling strength of $6 \mathrm{meV}$. Except for the spectra features, constant-height Kondo maps, reflecting the spin density distribution, also nicely agree with Hubbard model calculations (cf. Fig. 3b, e), further confirming our elucidation of $\mathrm{d} I / \mathrm{d} V$ spectra. These observations prove that breaking bipartite lattice symmetry will reverse the magnetic order between the two spins in diradical nBNGs. We also examined other dimer configurations, in support of the proposed exchange mechanism (cf. Supplementary Fig. 6).

Engineering exchange-coupling strength between two spins. The magnetic exchange-coupling strength can also be widely engineered in diradical NG dimers by tuning wavefunction (spin density) overlap of the zero-energy states ${ }^{18}$. The exchangecoupling strength can be expressed as $J=2 U \sum_{i}\left|\phi_{1}(i)\right|^{2}\left|\phi_{2}(i)\right|^{2}$, where $U$ denotes the on-site Coulomb repulsion and $\phi_{1} / \phi_{2}$ the wavefunction of the zero-energy state of up/down unit in the diradical dimer, respectively. Figure 4 a shows a BNG-BNG dimer with minimal spin density overlap. $\mathrm{d} I / \mathrm{d} V$ spectra taken on both BNG units exhibit a sharp Kondo resonance, similar to the features obtained on individual BNG monomers. This suggests that the two spins in this dimer has negligible exchange interaction due to negligible spin density overlap. We also examined another BNG-BNG dimer with maximum spin density overlap (cf. Fig. $4 \mathrm{~b}$ ). $\mathrm{d} I / \mathrm{d} V$ spectra reveal two steps located at $-42 \mathrm{mV}$ and 42
$\mathrm{mV}$, suggesting an exchange-coupling strength of $42 \mathrm{meV}$ (cf. Fig. 4d, e). The exchange-coupling interaction of different dimer configurations qualitatively agree with Hubbard model calculations (cf. Fig. 4f). These results demonstrate the wide tunability of magnetic exchange interaction in NGs, with implications for spintronic devices working above room temperature.

\section{Discussion}

We demonstrate an exotic approach of controlling magnetic exchange sign and strength in atomically precise diradical NG dimers by using combined non-contact atomic force microscopy, scanning tunneling microscopy/spectroscopy, as well as model calculations. The magnetic coupling sign transition is due to the spin density sign reversal at the connecting region by breaking the bipartite lattice symmetry of nanographene. As summarized in Table 1, we have calculated and checked all the dimer configurations (cf. details in Supplementary Figs. 5 and 6). Due to the negligible spin density overlap, all dimers in the $\boldsymbol{C} \mathbf{1}$ configuration host two spins with negligible magnetic coupling strength, which can be treated as two uncoupled spins at a finite temperature. In the $\boldsymbol{C} 2$ configuration, due to the revseral of spin density sign at the connecting region, both BNG-BNG and nBNG-BNG dimers host the ground state of $S=1$, while both $n B N G-n B N G$ and BNG-nBNG dimers host the ground state of $S=0$. In the $\boldsymbol{C 3}$ configuration, due to the strong spin density overlap, large magnetic exchange coupling up to $40 \mathrm{meV}$ has been achieved.

The nature behind this exchange mechanism is the minimization of on-site Coulomb repulsion in half-filling NGs. We demonstrated, by tailoring the spin density sign and spatial overlap, the magnetic coupling sign and strength can be widely tuned. Our method reported herein provides ample opportunities for designer above-room-temperature magnetic phases and 
a
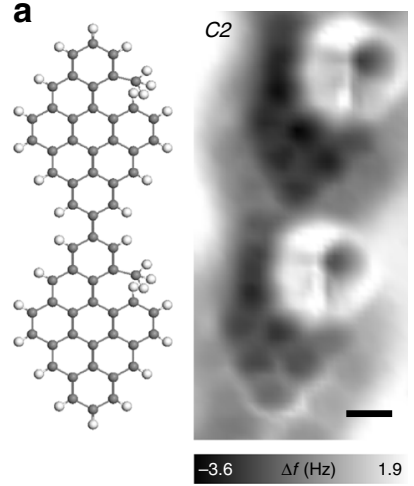

d

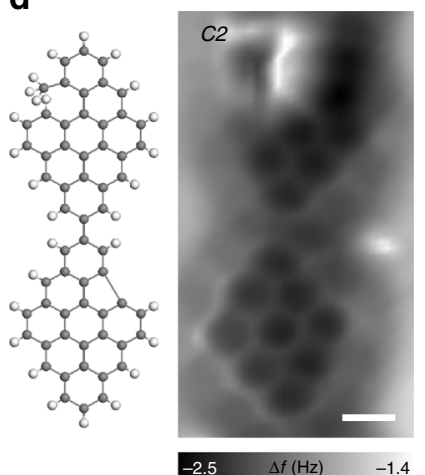

b

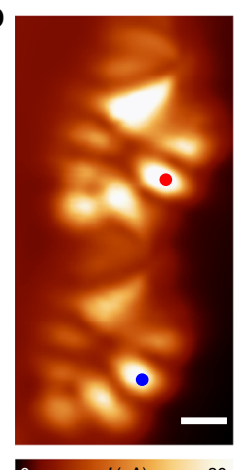

$\begin{array}{lll}0 & & \\ 0 & (\mathrm{pA}) \quad 30\end{array}$

e

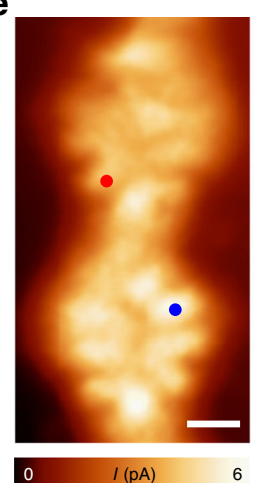

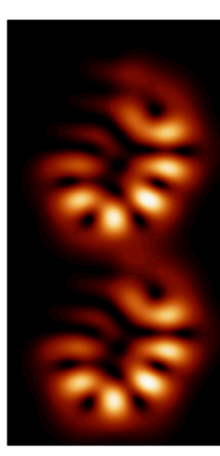

Low LDOS High

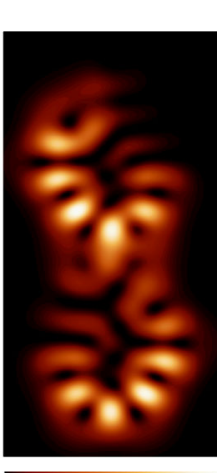

C
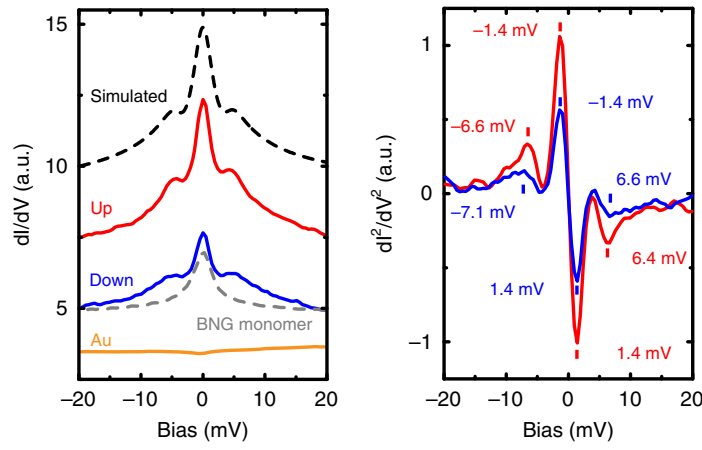

$f$

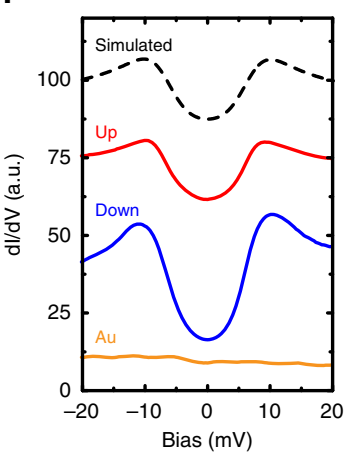

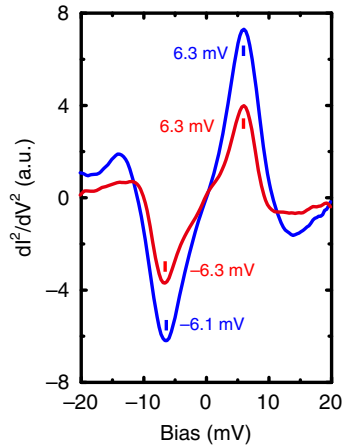

Fig. 3 Determining the spin order in diradical nanographene dimers by spin-flip spectroscopy. a Chemical structure and nc-AFM image (Resonant frequency: $29 \mathrm{KHz}$, Oscillation amplitude: $160 \mathrm{pm}$ ) of a BNG-BNG dimer. b Constant-height current image (Bias voltage: $1 \mathrm{mV}$ ) and simulated LDOS map of the dimer in $\mathbf{a} . \mathbf{c} \mathrm{d} l / \mathrm{d} V$ spectra (modulation: $0.1 \mathrm{mV}$, Temperature: $1.1 \mathrm{~K}$ ) and numerical calculated $\mathrm{d} l^{2} / \mathrm{d} V^{2}$ spectra taken on the two locations marked in $\mathbf{b}$. Three resonances have been detected, suggesting a ferromagnetic coupled ground state with $S=1$. The black dashed line is simulated line shape using a perturbative approach up to third order. d Chemical structure and nc-AFM image (Resonant frequency: $22 \mathrm{KHz}$, Oscillation amplitude: 140 pm) of a BNG-nBNG hetero-dimer. e Constant-height current image (Bias voltage: $1 \mathrm{mV}$ ) and simulated LDOS map of the dimer in $\mathbf{d}$. $\mathbf{f} \mathrm{d} l / \mathrm{dV}$ spectra and numerical

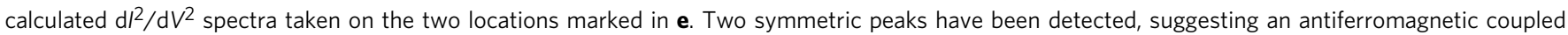
ground state with $S=0$. The black dashed line is simulated line shape using a perturbative approach up to third order. Scale bars: 0.3 nm.

functionalities in graphene nanomaterials, and could be extended to study many-body effects in low-dimensional $\pi$-electron quantum spin systems.

\section{Methods}

Sample preparation and characterization were carried out using a commercial lowtemperature Unisoku Joule-Thomson scanning probe microscopy under ultra-high vacuum conditions $\left(3 \times 10^{-10} \mathrm{mbar}\right)$. The high magnetic field spectroscopy measurements were carried out using a commercial Unisoku 1300 system. Au(111) single-crystal was cleaned by cycles of argon ion sputtering, and subsequently annealed to $800 \mathrm{~K}$ to get atomically flat terraces. Molecular precursors of 9,10-bis (4-bromo-2,6-dimethylphenyl)anthracene were thermally deposited on the clean $\mathrm{Au}(111)$ surface, and subsequently annealed to different temperatures to fabricate magnetic NGs (cf. Supplementary Fig. 1). Afterwards, the sample was transferred to a cryogenic scanner at $4.9 \mathrm{~K}(1.1 \mathrm{~K})$ for characterization. Carbon monoxide molecules are dosed onto the cold sample around $7 \mathrm{~K}\left(4 \times 10^{-9} \mathrm{mbar}, 2 \mathrm{~min}\right)$. To achieve ultra-high spatial resolution, CO molecule is picked up from Au surface to the apex of tungsten tip. A quartz tuning fork with resonant frequency of $31 \mathrm{KHz}$ has been used in nc-AFM measurements. A lock-in amplifier $(589 \mathrm{~Hz}, 0.1-0.5 \mathrm{mV}$ modulation) has been used to acquire $\mathrm{dI} / \mathrm{dV}$ spectra. The spectra were taken at 4.9 $\mathrm{K}$ unless otherwise stated.

The tight binding (TB) calculation of the STM images were carried out in the $C$ $2 P_{z}$-orbital description by numerically solving the Mean-Field-Hubbard Hamiltonian with nearest-neighbor hopping:

$$
\hat{H}_{\mathrm{MFH}}=\sum_{<i j>, \sigma}-t_{i j} c_{i, \sigma}^{+} c_{j, \sigma}^{-}+U \sum_{i, \sigma} n_{i, \sigma} n_{i, \bar{\sigma}}-U \sum_{i} n_{i, \uparrow} n_{i, \downarrow}
$$

with $t_{i j}$ is the nearest-neighbor hopping term depending on the bond length between $\mathrm{C}$ atoms and $c_{j, \sigma}^{+}$and $c_{j, \sigma}^{-}$denoting the spin selective $(\sigma=\{\uparrow, \downarrow\})$ creation and annihilation operators on the atomic site $i$ and $j, U$ the on-site Hubbard parameter (with $U=3.5 \mathrm{eV}$ ), $n_{i, \sigma}$ the number operator and $n_{i_{\sigma}}$ the mean occupation number at site $i$. A hopping integral of $2.7 \mathrm{eV}$ is used in our calculation unless otherwise stated. Numerically solving the model Hamiltonian yields the energy Eigenvalues $\mathrm{E}_{i}$ and the corresponding Eigenstates $\alpha_{i, j}$ (amplitude of state $i$ on site $j$ ) from which the wave functions are computed assuming Slater type atomic orbitals:

$$
\psi_{i}(\vec{r})=\sum_{j} \alpha_{i, j} \cdot\left(z-z_{j}\right) \exp \left(-\zeta\left|\vec{r}-\overrightarrow{r_{j}}\right|\right)
$$

with $\zeta=1.625$ a.u. for the carbon $2 P_{z}$ orbital. The charge density map $2 \rho(x, y)$ for a given energy range $\left[\varepsilon_{\min }, \varepsilon_{\max }\right]$ and height $z_{0}$ is then obtained by summing up the squared wave functions in this chosen energy range.

$$
\rho(x, y)=\sum_{i, \varepsilon_{i} \in\left[\varepsilon_{\min }, \varepsilon_{\max }\right]} \psi_{i}^{2}\left(x, y, z_{0}\right)
$$

Constant charge density maps are taken as a first approximation to compare with experimental STM images.

Spin-polarized DFT calculations were performed by using local spin-density approximation (LSDA) ${ }^{37}$ and generalized gradient approximation (GGA) ${ }^{38}$ method. The first-principles calculations were carried out with the Vienna Ab initio Simulation Package (VASP) ${ }^{39} . \mathrm{H}$ atoms were included to saturate dangling bonds. We add $10 \AA$ of vaccum in the direction perpendicular to the sheet and vacuum of $10 \AA$ in two orthogonal directions in plane to eliminate the periodic effect of the system. A cutoff of $700 \mathrm{eV}$ was used for convergence of the system for the short $\mathrm{C}-\mathrm{C}$ bond and $\mathrm{C}-\mathrm{H}$ bond. We optimized the geometric structure with the initial bond length of $\mathrm{C}-\mathrm{C}$ bond $1.42 \AA$ which was obtained from single sheet of graphene with LDA and GGA.

\section{Data availability}

The datasets generated and/or analyzed during this study are available from the corresponding author on reasonable request. 
a

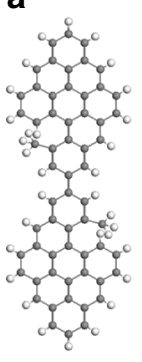

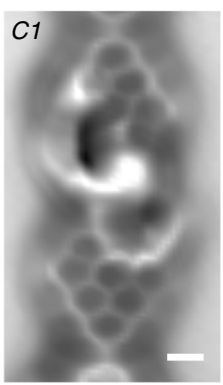

$\begin{array}{lll}-5.4 & \Delta f(\mathrm{~Hz}) \quad 5.2\end{array}$

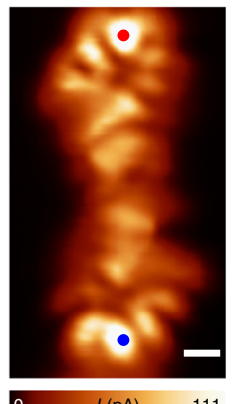

$\begin{array}{lll}0 & \text { I(PA) } & 111\end{array}$

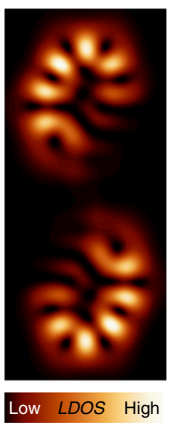

b

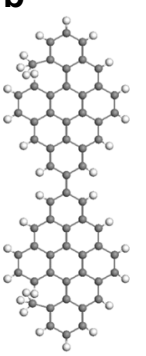

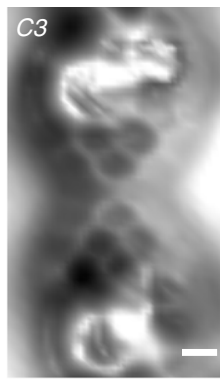

$-5.1 \quad \Delta f(\mathrm{~Hz}) \quad 3.5$

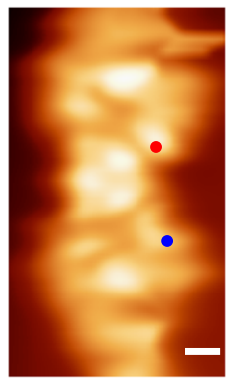

$\begin{array}{lll}0 & \text { I (nA) } & 1.2\end{array}$

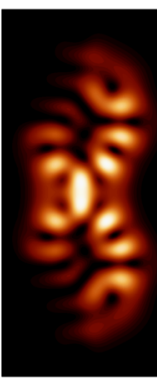

Low LDOS High
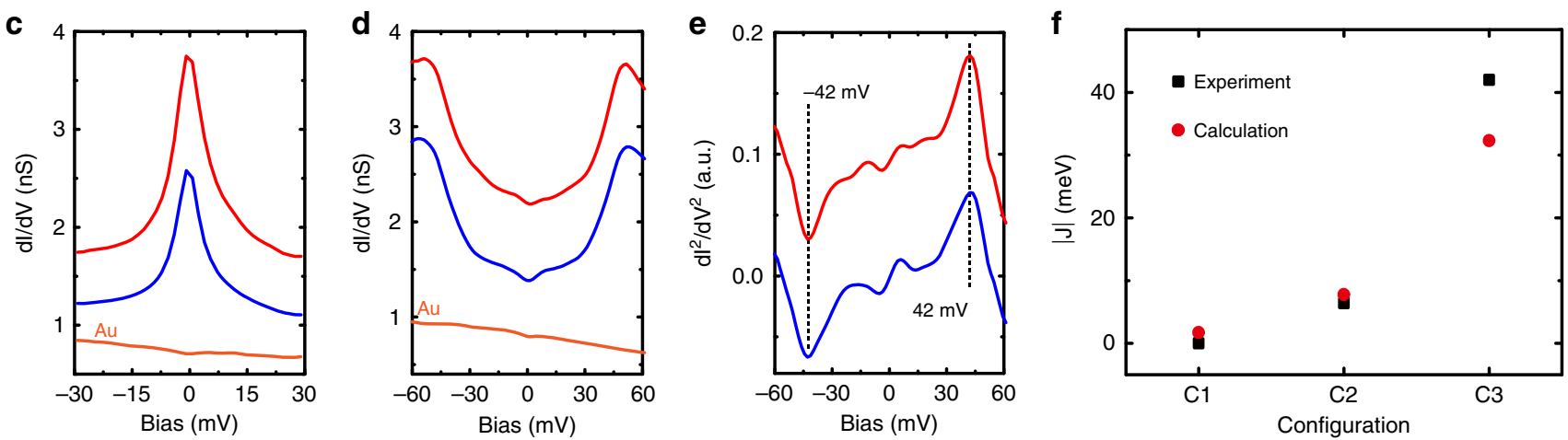

Fig. 4 Engineering exchange-interaction strength in diradical nanographene dimers. a From left to right: Chemical structure, nc-AFM image (Resonant frequency: $29 \mathrm{KHz}$, Oscillation amplitude: $160 \mathrm{pm}$ ), Constant-height current image (Bias voltage: $1 \mathrm{mV}$ ), and simulated LDOS map of a diradical nanographene dimer with minimum spin density overlap of the two spins. b From left to right: Chemical structure, nc-AFM image (Resonant frequency: $29 \mathrm{KHz}$, Oscillation amplitude: $160 \mathrm{pm}$ ), Constant-height current image (Bias voltage: $1 \mathrm{mV}$ ), and simulated LDOS map of a diradical nanographene dimer with maximum spin density overlap of the two spins. $\mathbf{c} \mathrm{d} / / \mathrm{d} V$ spectra spectra taken on the two locations marked in $\mathbf{a}$. The presence of Kondo resonances at each unit in the dimer suggest a negligible exchange interaction. $\mathbf{d}-\mathbf{e} \mathrm{d} l / \mathrm{d} V$ spectra and numerical calculated $\mathrm{d} / 2 / \mathrm{d} V^{2}$ spectra taken on the two locations marked in $\mathbf{b}$. Two symmetric peaks have been detected, suggesting an antiferromagnetic coupled ground state with exchange-interaction strength of $42 \mathrm{meV}$. f Summarized exchange-interaction strength of three dimer configurations C1-C3 marked in Figs. 3 and 4 . Scale bars: $0.3 \mathrm{~nm}$.

\section{Table 1 Summary of the ground state of all dimer configurations.}

\begin{tabular}{|c|c|c|c|c|}
\hline \multirow{2}{*}{$\begin{array}{l}\text { Ground state } \\
\text { Configuration }\end{array}$} & \multirow{2}{*}{$\begin{array}{l}\text { Uncoupled spins } \\
\text { C1 }\end{array}$} & \multicolumn{2}{|l|}{$\mathbf{S}=\mathbf{0}$} & \multirow{2}{*}{$\begin{array}{l}S=1 \\
C 2\end{array}$} \\
\hline & & $\mathrm{C2}$ & C3 & \\
\hline nBNG-nBNG & $0 \mathrm{meV}(0.15 \mathrm{meV})$ & $3.4 \pm 0.3 \mathrm{meV}(3.05 \mathrm{meV})$ & $35.8 \pm 0.4 \mathrm{meV}(28.80 \mathrm{meV})$ & - \\
\hline BNG-nBNG & $0 \mathrm{meV}(-0.95 \mathrm{meV})$ & $6.3 \pm 0.3 \mathrm{meV}(3.20 \mathrm{meV})$ & $42.9 \pm 0.4 \mathrm{meV}(30.50 \mathrm{meV})$ & - \\
\hline$n B N G-B N G$ & $0 \mathrm{meV}(-0.95 \mathrm{meV})$ & - & $42.9 \pm 0.4 \mathrm{meV}(30.50 \mathrm{meV})$ & $-5.9 \pm 0.3 \mathrm{meV}(-7.35 \mathrm{meV})$ \\
\hline
\end{tabular}

${ }^{\star \star}$ Exchange-coupling strength $J$ extracted from experimental (simulation) data. $\mathbf{C 1}, \mathbf{C 2}$, and $\mathbf{C 3}$ are the connecting configurations denoted in Figs. 3, 4 in the main paper.

\section{Code availability}

The tight-binding calculations were performed using a custom-made code on the MATLAB platform. Details of this tight-binding code can be obtained from the corresponding author on reasonable request.

Received: 4 August 2019; Accepted: 28 October 2020; Published online: 27 November 2020

\section{References}

1. Yazyev, O. V. Emergence of magnetism in graphene materials and nanostructures. Rep. Prog. Phys. 73, 056501 (2010).

2. Kuzemsky, A. L. Unconventional and exotic magnetism in carbon-based structures and related materials. Int. J. Mod. Phys. B 27, 1330007 (2013).

3. Lieb, E. H. Two theorems on the Hubbard model. Phys. Rev. Lett. 62, 1201-1204 (1989).
4. Fajtlowicz, S., John, P. E. \& Sachs, H. On maximum matchings and eigenvalues of benzenoid graphs. Croat. Chem. Acta 78, 195 (2005).

5. Yazyev, O. V. \& Helm, L. Defect-induced magnetism in graphene. Phys. Rev. B 75, 125408 (2007).

6. Yazyev, O. V. \& Katsnelson, M. I. Magnetic correlations at graphene edges: basis for novel spintronics devices. Phys. Rev. Lett. 100, 047209 (2008).

7. Palacios, J. J., Fernández-Rossier, J. \& Brey, L. Vacancy-induced magnetism in graphene and graphene ribbons. Phys. Rev. B 77, 195428 (2008).

8. Wang, W. L., Yazyev, O. V., Meng, S. \& Kaxiras, E. Topological frustration in graphene nanoflakes: magnetic order and spin logic devices. Phys. Rev. Lett. 102, 157201 (2009).

9. Makarova, T. L. Magnetic properties of carbon structures. Semiconductors 38, 615-638 (2004).

10. Coey, M. \& Sanvito, S. The magnetism of carbon. Phys. World 17, 33-37 (2004).

11. Son, Y.-W., Cohen, M. L. \& Louie, S. G. Half-metallic graphene nanoribbons. Nature 444, 347-349 (2006).

12. Pisani, L., Chan, J. A., Montanari, B. \& Harrison, N. M. Electronic structure and magnetic properties of graphitic ribbons. Phys. Rev. B 75, 064418 (2007). 
13. Ortiz, R., Lado, J. L., Melle-Franco, M. \& Fernández-Rossier, J. Engineering spin exchange in nonbipartite graphene zigzag edges. Phys. Rev. B 94, 094414 (2016).

14. Cui, P. et al. Carbon tetragons as definitive spin switches in narrow zigzag graphene nanoribbons. Phys. Rev. Lett. 116, 026802 (2016).

15. Fernández-Rossier, J. \& Palacios, J. J. Magnetism in Graphene Nanoislands. Phys. Rev. Lett. 99, 177204 (2007).

16. Clar, E. Polycyclic Hydrocarbons (Springer-Verlag Berlin Heidelberg, 1964).

17. Mishra, S. et al. Topological frustration induces unconventional magnetism in a nanographene. Nat. Nanotechnol. 15, 22-28 (2020).

18. Ortiz, R. et al. Exchange rules for diradical $\pi$-conjugated hydrocarbons. Nano Lett. 19, 5991-5997 (2019).

19. Sun, Z. \& Wu, J. Open-shell polycyclic aromatic hydrocarbons. J. Mater. Chem. 22, 4151-4160 (2012)

20. Wang, S. et al. Giant edge state splitting at atomically precise graphene zigzag edges. Nat. Commun. 7, 11507 (2016).

21. Ruffieux, P. et al. On-surface synthesis of graphene nanoribbons with zigzag edge topology. Nature 531, 489-492 (2016).

22. Tao, C. et al. Spatially resolving edge states of chiral graphene nanoribbons. Nat. Phys. 7, 616 (2011).

23. Li, J. et al. Single spin localization and manipulation in graphene open-shell nanostructures. Nat. Commun. 10, 200 (2019).

24. Mishra, S. et al. Synthesis and characterization of $\pi$-extended triangulene. $J$ Am. Chem. Soc. 141, 10621-10625 (2019).

25. Su, J. et al. Atomically precise bottom-up synthesis of $\pi$-extended [5] triangulene. Sci. Adv. 5, 7717 (2019).

26. Mishra, S. et al. Tailoring bond topologies in open-shell graphene nanostructures. ACS Nano 12, 11917-11927 (2018).

27. Pavliček, N. et al. Synthesis and characterization of triangulene. Nat. Nanotechnol. 12, 308-311 (2017).

28. Gonzalez-Herrero, H. et al. Atomic-scale control of graphene magnetism by using hydrogen atoms. Science 352, 437-441 (2016).

29. Mishra, S. et al. Topological defect-induced magnetism in a nanographene. J. Am. Chem. Soc. 142, 1147-1152 (2020).

30. Zheng, Y. et al. Engineering of magnetic coupling in nanographene. Phys. Rev. Lett. 124, 147206 (2020).

31. Kondo, J. Resistance minimum in dilute magnetic alloys. Prog. Theor. Phys. 32, 37-49 (1964)

32. Ternes, M., Heinrich, A. J. \& Schneider, W.-D. Spectroscopic manifestations of the Kondo effect on single adatoms. J. Phys. Condens. Matter 21, 053001 (2008).

33. Ormaza, M. et al. Efficient spin-flip excitation of a nickelocene molecule. Nano Lett. 17, 1877-1882 (2017).

34. Czap, G. et al. Probing and imaging spin interactions with a magnetic singlemolecule sensor. Science 364, 670-673 (2019).

35. Choi, D.-J. et al. Colloquium: atomic spin chains on surfaces. Rev. Mod. Phys. 91, 041001 (2019)

36. Ternes, M. Spin excitations and correlations in scanning tunneling spectroscopy. N. J. Phys. 17, 063016 (2015)

37. Hohenberg, P. \& Kohn, W. Inhomogeneous electron gas. Phys. Rev. 136, B864-B871 (1964)

38. Perdew, J. P., Burke, K. \& Ernzerhof, M. Generalized gradient approximation made simple. Phys. Rev. Lett. 77, 3865-3868 (1996).

39. Kresse, G. \& Furthmüller, J. Efficient iterative schemes for ab initio total-energy calculations using a plane-wave basis set. Phys. Rev. B 54, 11169-11186 (1996).

\section{Acknowledgements}

We acknowledge Prof. Markus Ternes for fruitful discussions. S.W. acknowledge financial support from National Natural Science Foundation of China (No. 11874258, 12074247) and Fok Yin Tung foundation. J.L. is grateful for the startup funding from the University of Hong Kong and the funding support from ITC to the SKL. This work is also supported by the Ministry of Science and Technology of China (Grants No. 2016YFA0301003, No. 2016YFA0300403), the National Natural Science Foundation of China (Grants No 11521404, No. 11634009, No. 11574202, No. 11874256, No. 11790313, No. 11674226, No. U1632102, No. 11674222, No. U1632272 and No. 11861161003), and the Strategic Priority Research Program of Chinese Academy of Sciences (Grant No. XDB28000000). We acknowledge SJTU HPC center for providing computational resources.

\section{Author contributions}

S.W. conceived the experiments, S.W., J.J., and X.F. supervised the project, Y.Q.Z., Y.Z., and G.W. performed the STM/STS and nc-AFM experiments. C.L. performed the meanfield Hubbard model calculations. C.X. and W.L. performed the DFT calculations. X.Y. performed the perturbation simulations. D.B. J.L. and X.F. synthesized the molecular precursor. D.G., Y.L., H.Z., and X.W. analyzed the data. S.W., Y.Q.Z., and C.L. wrote the paper. All authors discussed the results and implications and commented on the manuscript at all stages.

\section{Competing interests}

The authors declare no competing interests.

\section{Additional information}

Supplementary information is available for this paper at https://doi.org/10.1038/s41467 020-19834-2.

Correspondence and requests for materials should be addressed to X.F., S.W. or J.J.

Peer review information Nature Communications thanks the anonymous reviewer(s) for their contribution to the peer review of this work.

Reprints and permission information is available at http://www.nature.com/reprints

Publisher's note Springer Nature remains neutral with regard to jurisdictional claims in published maps and institutional affiliations.

Open Access This article is licensed under a Creative Common Attribution 4.0 International License, which permits use, sharing, adaptation, distribution and reproduction in any medium or format, as long as you give appropriate credit to the original author(s) and the source, provide a link to the Creative Commons license, and indicate if changes were made. The images or other third party material in this article are included in the article's Creative Commons license, unless indicated otherwise in a credit line to the material. If material is not included in the article's Creative Commons license and your intended use is not permitted by statutory regulation or exceeds the permitted use, you will need to obtain permission directly from the copyright holder. To view a copy of this license, visit http://creativecommons.org/ licenses/by/4.0/

(C) The Author(s) 2020 